\title{
A UNIFORM SPECTRAL GAP FOR CONGRUENCE COVERS OF A HYPERBOLIC MANIFOLD
}

\author{
DUBI KELMER AND LIOR SILBERMAN
}

\begin{abstract}
Let $G$ be $\mathrm{SO}(n, 1)$ or $\mathrm{SU}(n, 1)$ and let $\Gamma \subset G$ denote an arithmetic lattice. The hyperbolic manifold $\Gamma \backslash \mathcal{H}$ comes with a natural family of covers, coming from the congruence subgroups of $\Gamma$. In many applications, it is useful to have a bound for the spectral gap that is uniform for this family. When $\Gamma$ is itself a congruence lattice, there are very good bounds coming from known results towards the Ramanujan conjectures. In this paper, we establish an effective bound that is uniform for congruence subgroups of a non-congruence lattice.
\end{abstract}

\section{INTRODUCTION}

Let $G$ be a connected almost simple Lie group, let $K \subset G$ be a maximal compact subgroup and let $\Gamma \subset G$ be a lattice. We write $X=\Gamma \backslash G / K=\Gamma \backslash \mathcal{H}$ for the corresponding locally symmetric space, endowed with the hyperbolic Riemannian metric coming from the Killing form on $\operatorname{Lie}(G)$. Let $\triangle$ denote the (non-negative) Laplace-Beltrami operator on $\mathcal{H}$ and $X$, and let $\operatorname{Spec}(\Gamma)$ denote the point spectrum of this operator on $L^{2}(X)$ (that is, the set of eigenvalues with eigenfunctions in $\left.L^{2}(X)\right)$. Since $\Gamma$ is a lattice, the constant function is an eigenfunction and $0=\lambda_{0} \in \operatorname{Spec}(\Gamma)$. This eigenvalue is simple by the maximum principle, and we accordingly write $\lambda_{1}(\Gamma)$ for the smallest positive member of $\operatorname{Spec}(\Gamma)$.

We say that the lattice $\Gamma$ has property $(\tau)$ with respect to a family of finite index subgroups, if $\lambda_{1}\left(\Gamma^{\prime}\right)$ is uniformly bounded away from zero for all subgroups $\Gamma^{\prime} \subset \Gamma$ in this family. When $\Gamma$ is an arithmetic lattice, it has a natural family of finite-index subgroups, its congruence subgroups (defined below). We are interested in establishing property $(\tau)$ with respect to this family.

Remark 0.1. It was essentially shown by Brooks [Bro85] that the definition of property $(\tau)$ given above is equivalent to the condition that the trivial representation is isolated (in the Fell topology) from the other irreducible representations of $G$ occurring in $L^{2}\left(\Gamma^{\prime} \backslash G\right)$ for all $\Gamma^{\prime}$ in the family. The latter condition makes sense also for semisimple groups of higher rank, and this is the usual definition of property $(\tau)$. See the books [LZ03, Lub10] for more details.

When $G$ has property $(T)$ (the trivial representation is isolated in the full unitary dual), there is a uniform spectral gap for all locally symmetric spaces covered by $G / K$, in particular for the family of all finite-index subgroups of $\Gamma$. This is the case for groups $G$ with real rank greater than one, and also for the rank one groups $\operatorname{Sp}(n, 1)$ and $F_{4}^{-20}$.

In the remaining cases of $\mathrm{SO}(n, 1)$ and $\mathrm{SU}(n, 1)$, not only is there no uniform gap varying over all lattices, but the constructions of Randol [Ran74] and Selberg [Sel65] further show that arithmetic lattices never have property $(\tau)$ with respect to the family of all finite index subgroups.

Date: October 12, 2018. 
The starting point of our work is the fact that when $\Gamma$ is a congruence lattice (defined below), it has property $(\tau)$ with respect to its family of congruence subgroups. This was first established by Selberg for $\mathrm{SL}_{2}(\mathbb{Z}) \subset \mathrm{SL}_{2}(\mathbb{R}) \cong \mathrm{SO}(2,1)$, and later in many cases by Burger and Sarnak [BS91]. Finally Clozel [Clo03] obtained this in full generality. Moreover, for congruence lattices, there are uniform (and in many cases very good) explicit bounds for the spectral gap coming from known bounds towards the Generalized Ramanujan conjectures in the general linear group.

Much less is known when $\Gamma$ is arithmetic (but not congruence). For the special cases of $\mathrm{SO}(2,1)$ and $\mathrm{SO}(3,1)$, Sarnak and Xue [SX91] gave an elementary argument showing that any arithmetic lattice satisfies property $(\tau)$ with respect to its congruence subgroups. Their argument produces explicit bounds that are uniform for the part of the spectrum orthogonal to the spectrum of the original group. In this paper, we will generalize their method to obtain a similar result for all arithmetic lattices in $\mathrm{SO}(n, 1)$ and $\mathrm{SU}(n, 1)$. In particular, our result establishes property $(\tau)$ for the family of (most) congruence subgroups of a fixed arithmetic lattices. We note, however, that our result is no longer elementary: in order to establish property $(\tau)$ for arithmetic lattices, we rely on known property $(\tau)$ for congruence lattices.

Remark 0.2. The results on property $(\tau)$ for congruence lattices also apply for an (irreducible) congruence lattice in a semi-simple group of the form $G=\prod_{j} G_{j}$. Moreover, in this case the result is even stronger, and it implies that for any irreducible representation $\pi=\bigotimes_{j} \pi_{j}$ occurring in $L^{2}(\Gamma \backslash G)$ each factor $\pi_{j}$ is uniformly bounded away from the trivial representation. This stronger notion is referred to as a strong spectral gap; see [KS09] for details. In [Kel10], the first author extended the method of Sarnak and Xue to give a uniform bound for the strong spectral gap for congruence subgroups of a (potentially non-congruence) irreducible lattice in a product $\mathrm{SO}(2,1)^{d}$ for $d \geq 2$. It might be possible to use the results of this paper in order to obtain a similar result for a product of arbitrary rank one groups.

We now set the notation required for stating our main result. We parameterize the eigenvalue $\lambda$ in the standard form $\lambda_{s}=\rho^{2}-s^{2}$ with $s \in i \mathbb{R} \cup(0, \rho]$ and $\rho=\frac{n-1}{2}$ for $\operatorname{SO}(n, 1)$, $\rho=\frac{n}{2}$ for $\mathrm{SU}(n, 1)$. With this notation, we consider $\operatorname{Spec}(\Gamma)$ as a subset of $i \mathbb{R} \cup(0, \rho]$.

Let $F \subset \mathbb{R}$ denote a totally real number field with a fixed infinite place. Let $\mathbb{G} \subset \mathrm{GL}_{n}$ be a closed $F$-subgroup, such that $G=\mathbb{G}(\mathbb{R})$ is isomorphic to $\operatorname{SO}(n, 1)$ or $\mathrm{SU}(n, 1)$, while in all other infinite places $\mathbb{G}\left(F_{v}\right)$ is compact.

Let $\mathcal{O}_{F} \subset F$ denote the ring of integers of $F$ and set $\Delta=\mathbb{G}(F) \cap \mathrm{GL}_{n}\left(\mathcal{O}_{F}\right)$ (by abuse of notation we commonly write $\mathbb{G}\left(\mathcal{O}_{F}\right)$ for this group). Now, for any ideal $\mathfrak{q} \subset \mathcal{O}_{F}$, we have the principal congruence group

$$
\Delta(\mathfrak{q})=\{\gamma \in \Delta \mid \gamma \equiv I \quad(\bmod \mathfrak{q})\} .
$$

By definition, a lattice $\Gamma$ is a congruence group if it contains $\Delta(\mathfrak{q})$ for some $\mathfrak{q}$, and it is an arithmetic lattice if it is commensurable with $\Delta$. The definition of $\Delta$ above depends on the specific choice of embedding of $\mathbb{G}$ in a general linear group, but the commensurability class and the notion of a congruence subgroup are independent of this choice.

With these notation, the result of Clozel on property $(\tau)$ can be stated as follows: There is a positive constant $\alpha=\alpha(\mathbb{G})$ such that for all ideals $\mathfrak{q}$

$$
\operatorname{Spec}(\Delta(\mathfrak{q})) \subset i \mathbb{R} \cup(0, \rho-\alpha) \cup\{\rho\} .
$$


Remark 0.3. The Generalized Ramanujan Conjectures would imply that $\alpha=\min \{1, \rho\}$. For the orthogonal groups, the method of Burger and Sarnak [BS91], together with the results of $\left[\mathrm{KS} 03, \mathrm{BB} 10\right.$ ] on the automorphic spectrum of $\mathrm{GL}_{2}(\mathbb{A})$, imply that $\alpha \geq \frac{25}{64}$ for $\mathrm{SO}(2,1)$, and that $\alpha \geq \frac{25}{32}$ for $\mathrm{SO}(n, 1), n \geq 3$. These bounds also hold for certain arithmetic lattices in unitary groups. However, in general the best known bounds for the unitary groups are due to Clozel [Clo03, and come from lifting representations to $\mathrm{GL}_{n}(\mathbb{A})$ and using the bounds towards the Ramanujan Conjecture due to Luo, Rudnick and Sarnak [LRS99].

Fixing an arithmetic lattice $\Gamma$ we call the following its congruence subgroups:

$$
\Gamma(\mathfrak{q})=\Gamma \cap \Delta(\mathfrak{q}) .
$$

In what follows, we will assume without loss of generality (by replacing $\Gamma$ with $\Gamma(1)$ ) that $\Gamma$ is a finite index subgroup of $\Delta$. When $\mathfrak{q}^{\prime} \mid \mathfrak{q}$ we have $\Gamma(\mathfrak{q}) \subset \Gamma\left(\mathfrak{q}^{\prime}\right)$ so we can identify $L^{2}\left(\Gamma\left(\mathfrak{q}^{\prime}\right) \backslash \mathcal{H}\right)$ with the subspace of left- $\Gamma\left(\mathfrak{q}^{\prime}\right)$-invariant functions in $L^{2}(\Gamma(\mathfrak{q}) \backslash \mathcal{H})$. We write $L^{2}(\Gamma(\mathfrak{q}) \backslash \mathcal{H})_{\text {old }}$ for the sum of these subspaces as $\mathfrak{q}^{\prime}$ ranges over the (finite set) of ideals properly dividing $\mathfrak{q}$, and $L^{2}(\Gamma(\mathfrak{q}) \backslash \mathcal{H})_{\text {new }}$ for its orthogonal complement. Both spaces are preserved by the LaplaceBeltrami operator and we write $\operatorname{Spec}(\Gamma(\mathfrak{q}))_{\text {new }}$ for the set of eigenvalues in $L^{2}(\Gamma(\mathfrak{q}) \backslash \mathcal{H})_{\text {new }}$.

We can now state our main result.

Theorem 1. Let $\Gamma \subset \mathbb{G}\left(\mathcal{O}_{F}\right)$ be of finite index. There is an ideal $\mathfrak{d}=\mathfrak{d}(\Gamma)$, such that for any $\epsilon>0$ there is a constant $q_{0}=q_{0}(\Gamma, \epsilon)$ such that

$$
\operatorname{Spec}(\Gamma(\mathfrak{q}))_{\text {new }} \subseteq i \mathbb{R} \cup[0, \rho-\eta \alpha+\epsilon],
$$

for all ideals $\mathfrak{q}$ prime to $\mathfrak{d}$ of norm at least $q_{0}$. Here, $\alpha=\alpha(\mathbb{G})$ is as in (10.1) and $\eta=\eta(G)$ is an explicit constant given by

$$
\eta(G)=\left\{\begin{array}{cc}
\frac{4}{3 n(n+1)} & G \cong \mathrm{SO}(n, 1), 2 \leq n<6 \\
\frac{4(n-2)}{3 n(n+1)} & G \cong \mathrm{SO}(n, 1), n \geq 6 \\
\frac{2}{3(n+2)} & G \cong \mathrm{SU}(n, 1), n \geq 1
\end{array}\right.
$$

As an immediate corollary we get that $\Gamma$ satisfies property $(\tau)$ with respect to its congruence subgroups of levels prime to $\mathfrak{d}$. More precisely,

Corollary 0.1. There is a positive constant $\delta>0$ (depending on $\Gamma$ ) such that $\lambda_{1}(\Gamma(\mathfrak{q}))>\delta$ for all ideals $\mathfrak{q}$ prime to $\mathfrak{d}$.

Remark 0.4. Following the results of Breuillard, Green, and Tao [BGT10] and Pyber and Szabó [PS10], the work of Salehi Golsefidy and Varjú [SV10, Var10] (generalizing the results of Bourgain, Gamburd, and Sarnak [BGS10a, BGS10b]) also establishes property $(\tau)$ with respect to congruence subgroups of square free levels. However, this method does not produce an explicit bound for the spectral gap. In fact, [SV10, Var10] applies also when $\Gamma \subset \mathbb{G}\left(\mathcal{O}_{F}\right)$ is of infinite index, as long as it is geometrically finite and Zariski dense. See also [BV10] for similar results for $\Gamma \subset \mathrm{SL}_{n}(\mathbb{Z})$ and congruence subgroups of arbitrary level.

Before we explain the strategy of our proof, let us describe the original result of Sarnak and Xue in more detail. For any eigenvalue $\lambda$ we denote by $m(\lambda, \Gamma)$ the multiplicity of $\lambda$ in $\operatorname{Spec}(\Gamma)$. Using the Selberg trace formula, de George and Wallach dGW78 showed that for $\Gamma$ co-compact $m(\lambda, \Gamma) \lesssim \operatorname{vol}(\Gamma \backslash G)$ and that $m(\lambda, \Gamma)=o(\operatorname{vol}(\Gamma \backslash G))$ when $\lambda<\rho$. In [Xue91], Xue sharpened this result for congruence subgroups, showing that for $\lambda<\rho$,

$$
m(\lambda, \Gamma(\mathfrak{q})) \underset{3}{\lesssim} V(\mathfrak{q})^{1-\mu(\lambda)}
$$


where $V(\mathfrak{q})=[\Gamma: \Gamma(\mathfrak{q})]$ and $0<\mu(\lambda)<\frac{1}{n^{3}}$ is a small constant.

In [SX91], Sarnak and Xue conjectured that the correct bound should be

$$
m\left(\lambda_{s}, \Gamma(\mathfrak{q})\right) \lesssim \epsilon V(\mathfrak{q})^{\frac{\rho-s}{\rho}+\epsilon}
$$

They related this conjecture to a Diophantine conjecture, concerning the lattice point counting function

$$
N(\Gamma(\mathfrak{q}), T)=\{\gamma \in \Gamma(\mathfrak{q}) \mid d(\gamma o, o) \leq T\},
$$

where $o \in \mathcal{H}$ is the fixed point of $K$ and $d(x, y)$ is the hyperbolic distance. In particular, they conjectured that

$$
N(\Gamma(\mathfrak{q}), T) \lesssim \epsilon \frac{e^{2 \rho T(1+\epsilon)}}{V(\mathfrak{q})}+e^{\rho T},
$$

uniformly in $T$ and $\mathfrak{q}$, and showed that this conjecture implies the multiplicity bound (0.3).

For $\mathrm{SO}(2,1)$ and $\mathrm{SO}(3,1)$, they managed to establish the bound (0.4) by an elementary counting argument, thus proving (0.3) in these cases. Finally, for $\operatorname{SO}(2,1)$ (respectively $\mathrm{SO}(3,1))$ they showed that for eigenvalues in $\operatorname{Spec}(\Gamma(\mathfrak{q}))_{\text {new }}$, the spectral multiplicity $m(\lambda, \Gamma(\mathfrak{q}))$ is bounded below by $V(\mathfrak{q})^{\frac{1}{3}-\epsilon}$ (respectively $\left.V(\mathfrak{q})^{\frac{2}{3}-\epsilon}\right)$. Combining this bound with $(\underline{0.3})$, it followed that if $s<\rho-\frac{1}{6}$, then $\lambda_{s} \notin \operatorname{Spec}(\Gamma(\mathfrak{q}))_{\text {new }}$ when $V(\mathfrak{q})$ was sufficiently large.

In order to prove Theorem 1, we will follow the same general strategy. However, proving the Diophantine bound (0.4) in general by a direct counting argument seems out of reach. Instead we will take a different approach, using spectral theory and decay of matrix coefficients (similar arguments are used in [BKS10, GN09]). Our method does not give the conjectured bound (0.4). Instead, using (0.1) for the spectrum of congruence lattices, we get the following bound:

Theorem 2. $N(\Gamma(\mathfrak{q}), T) \lesssim \frac{e^{2 \rho T}}{V(\mathfrak{q})}+e^{(2 \rho-\alpha) T}$ uniformly in $T$ and $\mathfrak{q}$, where $\alpha$ is as in (0.1).

Using this weaker bound for the counting function, one gets the following intermediate bound for the multiplicities.

Theorem 3. $m\left(\lambda_{s}, \Gamma(\mathfrak{q})\right) \lesssim V(\mathfrak{q})^{\frac{\rho-s}{\alpha}}$ for $s \in(0, \rho)$.

Remark 0.5. For $G=\mathrm{SO}(2,1)$ or $\mathrm{SO}(3,1)$, the Selberg Conjecture reads $\alpha=\rho$. Assuming this, we recover the bounds of (0.3) and (0.4). In general, however, $\alpha$ is much smaller then $\rho$ even conjecturally, and Theorem 3 is trivial when $s<\rho-\alpha$ is small. That said, when $s$ is sufficiently close to $\rho$ our bound is comparable to the conjectured bound in (0.3) and suffices for our purposes.

The final ingredient we need is the lower bound for the multiplicities of new eigenvalues. For this we show

Theorem 4. There is an ideal $\mathfrak{d}$, depending on $\Gamma$, such that for all ideals $\mathfrak{q}$ prime to $\mathfrak{d}$, all $\lambda \in \operatorname{Spec}(\Gamma(\mathfrak{q}))_{\text {new }}$ satisfy

$$
m(\lambda, \Gamma(\mathfrak{q})) \gtrsim_{\epsilon} V(\mathfrak{q})^{\eta-\epsilon}
$$

where $\eta$ is as in (0.2).

Remark 0.6. If we consider only square-free ideals we can remove the condition that $\mathfrak{q}$ be prime to $\mathfrak{d}$ and at the same time slightly improve the bound, replacing $\eta$ by $\frac{3}{2} \eta$. 
Theorem 1 is now a direct consequence of Theorems 3 and 4 .

Remark 0.7. Using the conjectured bound (0.3) for the multiplicities, instead of the bound established in Theorem [3, would allow us to replace $\alpha \eta$ by $\rho \eta$ in Theorem 1. We note that when $n$ is large, this gives very strong bounds on the spectrum. In particular, for the orthogonal groups when $n \geq 16$, assuming (0.3) implies a spectral gap that is stronger then the best known bounds even in the congruence case.

Acknowledgements. We thank P. Varjú for explaining his results. The first author was partially supported by the NSF grant DMS-1001640. The second author was partially supported by an NSERC Discovery Grant.

\section{BACKGROUND AND NOTATION}

We write $A \lesssim B$ or $A=O(B)$ to indicate that $A \leq c B$ for some constant $c$. If we wish to emphasize that constant depends on some parameters we use subscripts, for example $A \lesssim_{\epsilon} B$. Note that all the implied constants in this paper may depend on the group $\mathbb{G}$, which we consider fixed. Sometimes they only depend on the group $G$; in that case they are expressed via the number $n$. We also write $A \asymp B$ to indicate that $A \lesssim B \lesssim A$. Finally, the cardinality of a finite set $S$ will be denoted $\# S$ or $|S|$.

1.1. Lie theory. $G$ will be an almost simple Lie group of real rank $1 ; K \subset G$ will be a maximal compact subgroup so that $\mathcal{H}=G / K$ is a rank one symmetric space. The Killing form on the Lie algebra $\mathfrak{g}$ of $G$ induces a $G$-invariant Riemannian structure on $\mathcal{H}$ with the Riemannian measure $d x$. In particular, we will consider the cases of $G=\mathrm{SO}(n, 1)$ and $G=\mathrm{SU}(n, 1)$ corresponding to real and complex hyperbolic spaces respectively.

Let $G=N A K$ be an Iwasawa decomposition of $G$, with $A$ a maximal diagonalizable subgroup and $N$ a maximal unipotent subgroup. Let $\mathfrak{a}$ denote the Lie algebra of $A$ and let $\mathfrak{a}_{\mathbb{C}}^{*}$ denote its complexified dual (one dimensional since $G$ is of rank one). Fix a positive Weyl chamber, denote by $\rho$ the half sum of the positive roots, and fix a positive $X \in \mathfrak{a}$ of norm one with respect to the Killing form. We now identify $\mathfrak{a}_{\mathbb{C}}^{*}$ with $\mathbb{C}$ via their values at $X$ and, slightly abusing notation, write $\rho=\rho(X) \in \mathbb{R}$ so that $\rho=\frac{n-1}{2}$ in the case of $\operatorname{SO}(n, 1)$ and $\rho=\frac{n}{2}$ in the case of $\mathrm{SU}(n, 1)$.

We normalize the Haar measure $d k$ of $K$ to have total mass 1 and the Haar measure $d g$ on $G$ so that it projects to $d x$ on $\mathcal{H}=G / K$. We have the Cartan decomposition $G=K A^{+} K$ with $A^{+}$the closure of the positive Weyl chamber. Let $X \in \mathfrak{a}$ be as above, then any $g \in G$ can be expressed in the form $g=k_{1}(g) \exp (t(g) X) k_{2}(g)$ with $k_{j} \in K$ and $t(g)=d(g o, o) \in \mathbb{R}^{+}$. The Haar measure with respect to this decomposition is given by $d g=D(t) d k_{1} d t d k_{2}$ with $D(t) \asymp e^{2 \rho t}$ (see [Kna86, Proposition 5.28]).

1.2. Spectral theory. Denote by $\hat{G}$ the unitary dual of $G$ and by $\hat{G}^{1}$ the spherical dual. We parameterize $\hat{G}^{1}$ by $\mathfrak{a}_{\mathbb{C}}^{*} / W$ where $W$ is the Weyl group. With this parametrization the tempered representations lie in $i \mathfrak{a}_{\mathbb{R}}^{*}$ and the non-tempered representations are contained in $(0, \rho]$. We use the notation

$$
\hat{G}^{1}=\left\{\pi_{s} \mid s \in i \mathbb{R}^{+} \cup[0, \rho]\right\},
$$

where the representations $\pi_{s}, s \in i \mathbb{R}^{+}$are the (tempered) principal series representations, the representations $\pi_{s}, s \in(0, \rho)$ are the (non-tempered) complementary series, and $\pi_{\rho}$ is the trivial representation. 
We recall the relation between Laplace eigenvalues and irreducible representations. For this let $\Omega$ be the Casimir operator of $G, \Omega$ acts on any irreducible representation $V_{\pi}$ by scalar multiplication $\Omega v+\lambda(\pi) v=0$. We may normalize $\Omega$ so that the restriction of $\Omega$ to the space of right- $K$-invariant functions on $G$ coincides with the Laplace-Beltrami operator $\triangle$ on $\mathcal{H}=G / K$. With this normalization we have $\lambda\left(\pi_{s}\right)=\rho^{2}-s^{2}$.

1.3. Decay of matrix coefficients. For each $s \in i \mathbb{R}^{+} \cup[0, \rho]$ consider the spherical function defined by

$$
\phi_{s}(g)=\left\langle\pi_{s}(g) v, v\right\rangle
$$

where $v \in V_{\pi_{s}}$ is the normalized spherical vector. The asymptotic behavior of this function is well-known. For $s \in(0, \rho]$ the function $\phi_{s}$ is positive and decays like

$$
\phi_{s}(\exp (t X)) \asymp C_{s} e^{(s-\rho) t} .
$$

For $s \in i \mathbb{R}, \phi_{s}$ is oscillatory and decays like

$$
\left|\phi_{s}(\exp (t X))\right| \lesssim t e^{-\rho t}
$$

As a consequence, we have the following bounds on general matrix coefficients: Let $\pi$ denote a unitary representation of $G$ on a Hilbert space $V_{\pi}$ such that all non-trivial non-tempered $\pi_{s}$ weakly contained in $V_{\pi}$ satisfy $s \in[0, \rho-\alpha]$. Then for any smooth spherical vector $v \in V_{\pi}$,

$$
|\langle\pi(g) v, v\rangle| \leq C(\alpha)\|v\|^{2} e^{-\alpha t(g)} .
$$

\section{Proof of Theorem 2}

Since $\Gamma(\mathfrak{q}) \subset \Delta(\mathfrak{q})$, we have $N(\Gamma(\mathfrak{q}) ; T) \leq N(\Delta(\mathfrak{q}) ; T)$, and it is sufficient to bound the latter, that is to show that

$$
\sharp\{\gamma \in \Delta(\mathfrak{q}) \mid t(\gamma) \leq T\} \lesssim \frac{e^{2 \rho T}}{V(\mathfrak{q})}+e^{(2 \rho-\alpha) T} .
$$

It is easier to obtain this bound in a smooth form. Accordingly for $g, h \in G$ let

$$
N(\Delta(\mathfrak{q}), g, h ; T)=\sharp\left\{\gamma \in \Delta(\mathfrak{q}) \mid t\left(h^{-1} \gamma g\right) \leq T\right\} .
$$

Fix a fundamental domain $\mathcal{F}_{1}$ for $\Delta \backslash G$ (containing the identity) and let $B \subset \mathcal{F}_{1}$ be a small fixed ball. Let $\psi$ denote a smooth positive bi- $K$ invariant function on $G$ of total mass one that is supported on $B$. Consider the averaged counting function

$$
\Psi(T)=\int_{B} \int_{B} N(\Delta(\mathfrak{q}), g, h ; T) \psi(g) \psi(h) d g d h .
$$

Since $\psi$ is supported inside a fundamental domain $\mathcal{F}_{1}$ it is also supported in a fundamental domain $\mathcal{F}_{\mathfrak{q}}$ for $\Delta(\mathfrak{q}) \backslash G$. Let $\tilde{\psi}(g)=\sum_{\gamma \in \Gamma(\mathfrak{q})} \psi(\gamma g)$ denote the periodized function on $\Delta(\mathfrak{q}) \backslash G$.

\section{Lemma 2.1.}

$$
\Psi(T)=\int_{G} \mathbb{1}_{T}(g)\left\langle R_{\mathfrak{q}}(g) \tilde{\psi}, \tilde{\psi}\right\rangle d g,
$$

where $\mathbb{1}_{T}(g)$ is the indicator function of the set $\{g \in G \mid t(g) \leq T\}$ and $R_{\mathfrak{q}}$ is the right regular representation of $G$ on $L^{2}(\Delta(\mathfrak{q}) \backslash G)$. 
Proof. Since $\psi$ is supported on $B \subset \mathcal{F}_{\mathfrak{q}}$ we can replace the integral over $B \times B$ in (2.2) with an integral over $\mathcal{F}_{\mathfrak{q}} \times \mathcal{F}_{\mathfrak{q}}$. Writing the counting function as

$$
N(\Delta(\mathfrak{q}), g, h ; T)=\sum_{\gamma \in \Gamma(\mathfrak{q})} \mathbb{1}_{T}\left(h^{-1} \gamma g\right),
$$

we get

$$
\Psi(T)=\int_{\mathcal{F}_{q}} \int_{\mathcal{F}_{q}}\left(\sum_{\gamma \in \Gamma(\mathfrak{q})} \mathbb{1}_{T}\left(h^{-1} \gamma g\right)\right) \tilde{\psi}(g) \tilde{\psi}(h) d g d h .
$$

Changing variables $g \mapsto \gamma^{-1} g$ and using the $\Gamma(\mathfrak{q})$-invariance of $\tilde{\psi}$ we get

$$
\begin{aligned}
\Psi(T) & =\int_{\mathcal{F}_{\mathfrak{q}}}\left(\sum_{\gamma \in \Gamma(\mathfrak{q})} \int_{\gamma F_{\mathfrak{q}}} \mathbb{1}_{T}\left(h^{-1} g\right) \tilde{\psi}(g) d g\right) \tilde{\psi}(h) d h \\
& =\int_{\mathcal{F}_{\mathfrak{q}}}\left(\int_{G} \mathbb{1}_{T}\left(h^{-1} g\right) \tilde{\psi}(g) d g\right) \tilde{\psi}(h) d h .
\end{aligned}
$$

Making one more change of variables $g \mapsto h g$ and changing the order of integration we get

$$
\begin{aligned}
\Psi(T) & =\int_{\mathcal{F}_{\mathfrak{q}}}\left(\int_{G} \mathbb{1}_{T}(g) \tilde{\psi}(h g) d g\right) \tilde{\psi}(h) d h \\
& =\int_{G} \mathbb{1}_{T}(g)\left(\int_{\mathcal{F}_{\mathfrak{q}}} \tilde{\psi}(h g) \tilde{\psi}(h) d h\right) d g \\
& =\int_{G} \mathbb{1}_{T}(g)\left\langle R_{\mathfrak{q}}(g) \tilde{\psi}, \tilde{\psi}\right\rangle d g .
\end{aligned}
$$

We can now use the results about decay of matrix coefficients to bound $\Psi(T)$ :

Lemma 2.2. $\Psi(T) \lesssim \frac{e^{2 \rho T}}{V(\mathfrak{q})}+e^{(\rho-\alpha) T}$.

Proof. Decompose $\tilde{\psi}=\psi_{0}+\psi^{\perp}$, with $\psi_{0}=\frac{1}{\operatorname{vol}(\Delta(\mathfrak{q}) \backslash G)} 1$ the projection of $\tilde{\psi}$ onto the constant function and $\psi_{0}^{\perp} \in L_{0}^{2}(\Delta(\mathfrak{q}) \backslash G)$ its orthogonal complement. We then have

$$
\left\langle R_{\mathfrak{q}}(g) \tilde{\psi}, \tilde{\psi}\right\rangle=\left\|\psi_{0}\right\|^{2}+\left\langle R_{\mathfrak{q}}(g) \psi_{0}^{\perp}, \psi_{0}^{\perp}\right\rangle .
$$

Since $\Delta(\mathfrak{q})$ is a congruence group, all non-tempered $\pi_{s} \in \hat{G}^{1}$ (weakly) contained in $L_{0}^{2}(\Delta(\mathfrak{q}) \backslash G)$ satisfy $s \in[0, \rho-\alpha)$. Consequently, we get that

$$
\left\langle R_{\mathfrak{q}}(g) \psi^{\perp}, \psi^{\perp}\right\rangle \lesssim\left\|\psi^{\perp}\right\|^{2} e^{-\alpha t(g)}
$$

We have $\left\|\psi_{0}\right\|^{2}=\frac{1}{\operatorname{vol}(\Delta(\mathfrak{q}) \backslash G)} \asymp \frac{1}{V(\mathfrak{q})}$ and we can bound $\left\|\psi^{\perp}\right\|^{2} \leq\|\tilde{\psi}\|^{2}=\int_{B}|\psi(g)|^{2} d g$ which is independent on $q$. Plugging this in (2.3) gives

$$
\Psi(T) \lesssim \int_{0}^{T}\left(\frac{1}{V(\mathfrak{q})}+e^{-\alpha t}\right) e^{2 \rho t} d t \lesssim \frac{e^{2 \rho T}}{V(\mathfrak{q})}+e^{(2 \rho-\alpha) T}
$$


We can now conclude the proof of (2.1), and hence of Theorem 2. Since on average

$$
\Psi(T)=\int_{B} \int_{B} N(T, \Delta(\mathfrak{q}) ; g, h) \psi(g) \psi(h) d g d h \lesssim \frac{e^{2 \rho T}}{V(\mathfrak{q})}+e^{(2 \rho-\alpha) T},
$$

there is a point $(g, h) \in B \times B$ for which

$$
N(T, \Delta(\mathfrak{q}) ; g, h) \lesssim \frac{e^{2 \rho T}}{V(\mathfrak{q})}+e^{(2 \rho-\alpha) T}
$$

Now, since $B$ is compact, there is some $\delta=\delta(B)$ (not depending on $\mathfrak{q}$ ) such that for any $h, g \in B$

$$
t(\gamma)-\delta \leq t\left(h^{-1} \gamma g\right) \leq t(\gamma)+\delta
$$

Replacing $T$ by $T \pm \delta$ (which only affects the implied constant) we get (2.1).

\section{Proof of Theorem 3}

Theorem 3 follows from Theorem 2 by the same arguments as in the proof of [SX91, Theorem 3] (and [HK93] for the non-compact case). For the sake of completeness we include the details below.

Fix an eigenvalue $\lambda=\rho^{2}-s^{2}$ and let $V_{\lambda}(\Gamma(\mathfrak{q}))$ denote the corresponding eigenspace. Fix an orthonormal basis $\psi_{1}, \ldots, \psi_{m}$ for $V_{\lambda}(\Gamma(\mathfrak{q}))$ and consider the Bergman function

$$
B(x ; \mathfrak{q}, \lambda)=\sum_{j=1}^{m}\left|\psi_{j}(x)\right|^{2} .
$$

Note that

$$
B(x ; \mathfrak{q}, \lambda)=\sup _{\psi \in V_{\lambda}(\Gamma(\mathfrak{q}))} \frac{|\psi(x)|^{2}}{\|\psi\|^{2}},
$$

is independent on the choice of basis. Since the left action of $\Gamma$ preserves $V_{\lambda}(\Gamma(\mathfrak{q}))$, the function $B(x ; \mathfrak{q}, \lambda)$ is $\Gamma$ invariant and

$$
\int_{\mathcal{F}_{1}} B(x ; \mathfrak{q}, \lambda) d x=\frac{m(\lambda, \mathfrak{q})}{V(\mathfrak{q})} .
$$

In the case of non-compact $\mathcal{F}_{1}$, [HK93, Lemma 2.1] obtains a compact neighborhood of the identity $\mathcal{F}_{0} \subset \mathcal{F}_{1}$ (independent of $\mathfrak{q}$ ) such that

$$
\int_{\mathcal{F}_{0}} B(x ; \mathfrak{q}, \lambda) d x \gtrsim \frac{m(\lambda, \mathfrak{q})}{V(\mathfrak{q})} .
$$

uniformly for $\lambda \leq \rho^{2}-1$, where the implied constant depends only on $\mathcal{F}_{0}$.

Let $f(g)=\mathbb{1}_{T}(g) \phi_{s}(g)$ with $\mathbb{1}_{T}$ as above and $\phi_{s}$ the spherical function defined in (1.1). Let $F=f * \check{f}$ where $\check{f}(g)=\bar{f}\left(g^{-1}\right)$. By [SX91, Lemma 2.1] the function $F(g)$ is bi- $K$ invariant and satisfies

$$
F(g) \lesssim\left\{\begin{array}{cl}
e^{2 s T} e^{-\rho t(g)} & \text { if } t(g) \leq 2 T \\
0 & \text { if } t(g)>2 T
\end{array}\right.
$$

Its spherical transform is given by $\hat{F}(s)=|\hat{f}(s)|^{2}$ where

$$
\hat{f}(s)=\int_{G} \mathbb{1}_{T}(g)\left|\phi_{s}(g)\right|^{2} d g \asymp \int_{8}^{T} e^{2 \rho t} e^{2(s-\rho) t} d t \asymp e^{2 s T},
$$


so that $\hat{F}(s) \asymp e^{4 s T}$.

Identifying $\Gamma(\mathfrak{q}) \backslash \mathcal{H}=\Gamma(\mathfrak{q}) \backslash G / K$ and using the pre-trace formula we have

$$
\sum_{\gamma \in \Gamma(\mathfrak{q})} F\left(x^{-1} \gamma x\right)=\sum_{k} \hat{F}\left(s_{k}\right)\left|\psi_{k}(x)\right|^{2}+\mathcal{E},
$$

where the sum on the right is over a basis of Laplacian eigenfunctions in $L^{2}(\Gamma(\mathfrak{q}) \backslash \mathcal{H})=$ $L^{2}(\Gamma(\mathfrak{q}) \backslash G / K)$ with eigenvalue $\lambda_{k}=\rho^{2}-s_{k}^{2}$ and $\mathcal{E}$ corresponds to the contribution of the continuous spectrum (this is a finite sum of integrals of $\hat{F}$ against Eisenstein series). From positivity of $\hat{F}$ we can bound

$$
\hat{F}(s) B\left(x ; \mathfrak{q}, \lambda_{s}\right) \leq \sum_{\gamma \in \Gamma(\mathfrak{q})} F\left(x^{-1} \gamma x\right) .
$$

From compactness of $\mathcal{F}_{0}$ there is $\delta$ depending only on $\mathcal{F}_{0}$ (not on $\mathfrak{q}$ ) such that

$$
t(\gamma)-\delta \leq t\left(x^{-1} \gamma x\right) \leq t(\gamma)+\delta
$$

for all $x \in \mathcal{F}_{0}$. We thus get that

$$
\begin{aligned}
\hat{F}(s) B\left(x ; \mathfrak{q}, \lambda_{s}\right) & \lesssim e^{2 s T} \sum_{\substack{\gamma \in \Gamma(\mathfrak{q}) \\
t\left(x^{-1} \gamma x\right) \leq 2 T}} e^{-\rho t\left(x^{-1} \gamma x\right)} \\
& \lesssim e^{2 s T} \sum_{\substack{\gamma \in \Gamma(\mathfrak{q}) \\
t(\gamma) \leq 2 T}} e^{-\rho t(\gamma)}
\end{aligned}
$$

uniformly on $\mathcal{F}_{0}$. Integrating by parts, and using Theorem 2 we get

$$
\begin{aligned}
\hat{F}(s) B\left(x ; \mathfrak{q}, \lambda_{s}\right) & \lesssim e^{2 s T} \int^{2 T} e^{-\rho t} N(\Gamma(\mathfrak{q}) ; t) d t \\
& \lesssim e^{2 s T} \int^{2 T} e^{-\rho t}\left(\frac{e^{2 \rho t}}{V(\mathfrak{q})}+e^{(2 \rho-\alpha) t}\right) d t \\
& \lesssim e^{2 s T}\left(\frac{e^{2 \rho T}}{V(\mathfrak{q})}+e^{2(\rho-\alpha) T}\right) .
\end{aligned}
$$

Dividing by $\hat{F}(s) \asymp e^{4 s T}$ and integrating over $\mathcal{F}_{0}$ we get

$$
\frac{m\left(\lambda_{s}, \Gamma(\mathfrak{q})\right)}{V(\mathfrak{q})} \lesssim \int_{\mathcal{F}_{0}} B\left(x ; \mathfrak{q}, \lambda_{s}\right) d x \lesssim e^{-2 s T}\left(\frac{e^{2 \rho T}}{V(\mathfrak{q})}+e^{2(\rho-\alpha) T}\right)
$$

Now multiply by $V(\mathfrak{q})$ and take $T=\frac{\log (V(\mathfrak{q}))}{2 \alpha}$ to get $m\left(\lambda_{s}, \Gamma(\mathfrak{q})\right) \lesssim V(\mathfrak{q})^{\frac{(\rho-s)}{\alpha}}$.

\section{Proof of Theorem 4 .}

The lower bound for the multiplicities of eigenvalues in $\operatorname{Spec}(\Gamma(\mathfrak{q}))_{\text {new }}$ follows from a lower bound on the dimensions of irreducible representations of the finite groups $\Gamma(\mathfrak{q}) \backslash \Gamma$. We say that a representation of $\Gamma(\mathfrak{q}) \backslash \Gamma$ is new if it does not factor through one of the quotients $\Gamma\left(\mathfrak{q}^{\prime}\right) \backslash \Gamma$, for any proper divisor $\mathfrak{q}^{\prime}$ of $\mathfrak{q}$; equivalently, we say that a representation of $\Gamma$ is of level $\mathfrak{q}$ if it factors through a new representation of $\Gamma(\mathfrak{q}) \backslash \Gamma$. 
Since $\Gamma(\mathfrak{q})$ is normal in $\Gamma$, the left action of $\Gamma$ preserves $L^{2}(\Gamma(\mathfrak{q}) \backslash \mathcal{H})$ and commutes with the Laplacian. We thus get a representation of $\Gamma(\mathfrak{q}) \backslash \Gamma$ on each eigenspace $V_{\lambda}(\Gamma(\mathfrak{q}))$. Moreover, $\lambda \in \operatorname{Spec}(\Gamma(\mathfrak{q}))$ belongs to $\operatorname{Spec}(\Gamma(\mathfrak{q}))_{\text {new }}$ iff there is at least one invariant subspace in $V_{\lambda}(\Gamma(\mathfrak{q}))$ on which $\Gamma / \Gamma(\mathfrak{q})$ acts via a new representation. Consequently, we can reduce Theorem 4 to the following result regarding the irreducible representations of $\Gamma(\mathfrak{q}) \backslash \Gamma$.

Proposition 4.1. There is an ideal $\mathfrak{d}$ depending on $\Gamma$ such that all irreducible representations of level $\mathfrak{q}$ prime to $\mathfrak{d}$ satisfy

$$
\operatorname{dim}(\rho) \gtrsim \epsilon(\mathfrak{q})^{\eta-\epsilon}
$$

4.1. Reduction to prime powers. The Strong Approximation Theorem of Weisfeiler [Wei84, Thm. 1] (together with the Borel Density Theorem) show that there exists an ideal $\mathfrak{d}_{1}$ in $\mathcal{O}_{F}$ and a group scheme $\mathbf{G}$ defined over the localization of $\mathcal{O}_{F}$ away from $\mathfrak{d}_{1}$ such that $\mathbf{G}_{F}=\mathbb{G}$, and such that for all $\mathfrak{q}$ prime to $\mathfrak{d}_{1}$ there exists an isomorphism

$$
\Gamma(\mathfrak{q}) \backslash \Gamma \cong \mathbf{G}\left(\mathcal{O}_{F} / \mathfrak{q}\right) \cong \prod_{j=1}^{\omega(\mathfrak{q})} \mathbf{G}\left(\mathcal{O}_{F} / \mathfrak{p}_{j}^{r_{j}}\right)
$$

where $\mathfrak{q}=\prod_{j} \mathfrak{p}_{j}^{r_{j}}$ is the prime factorization of $\mathfrak{q}$. It immediately follows that

$$
V(\mathfrak{q})=|\Gamma(\mathfrak{q}) \backslash \Gamma|=\prod_{j=1}^{\omega(\mathfrak{q})}\left|\mathbf{G}\left(\mathcal{O}_{F} / \mathfrak{p}_{j}^{r_{j}}\right)\right|
$$

Further, any irreducible representation $\rho$ of $\mathbf{G}\left(\mathcal{O}_{F} / \mathfrak{q}\right)$ is isomorphic to a tensor product of irreducible representations $\rho_{j}$ of $\mathbf{G}\left(\mathcal{O}_{F} / \mathfrak{p}_{j}^{r_{j}}\right)$. We thus also have

$$
\operatorname{dim}(\rho)=\prod_{j=1}^{\omega(\mathfrak{q})} \operatorname{dim}\left(\rho_{j}\right)
$$

Finally, $\rho$ is of level $\mathfrak{q}$ if and only if the $\rho_{j}$ are of levels $\mathfrak{p}_{j}^{r_{j}}$. Since $\left|\mathbf{G}\left(\mathcal{O}_{F} / \mathfrak{p}^{r}\right)\right| \asymp\left|\mathrm{k}_{\mathfrak{p}}\right|^{r \operatorname{dim}(G)}$ and the number $\omega(\mathfrak{q})$ of prime divisors of $\mathfrak{q}$ satisfies $2^{\omega(\mathfrak{q})} \lesssim_{\epsilon}\left|\mathcal{O}_{F} / \mathfrak{q}\right|^{\epsilon}$, we can reduce Proposition 4.1 to the following result regarding the irreducible new representations of $\mathbf{G}\left(\mathcal{O}_{F} / \mathfrak{p}^{r}\right)$.

Proposition 4.2. There is an ideal $\mathfrak{d}$ (contained in $\mathfrak{d}_{1}$ ) such that for all prime ideals $\mathfrak{p}$ not dividing $\mathfrak{d}$, any new representation $\rho$ of $\mathbf{G}\left(\mathcal{O}_{F} / \mathfrak{p}^{r}\right)$ satisfies

$$
\operatorname{dim} \rho \gtrsim\left|\mathrm{k}_{\mathfrak{p}}\right|^{\frac{2 r e}{3}}
$$

where $\mathrm{k}_{\mathfrak{p}}=\mathcal{O}_{F} / \mathfrak{p}$ is the corresponding residue field and

$$
e=e(\mathbf{G})=\frac{3 \eta \operatorname{dim}(\mathbf{G})}{2}=\left\{\begin{array}{cc}
1 & \mathbf{G}(\mathbb{R}) \cong \mathrm{SO}(n, 1), n<6 \\
n-2 & \mathbf{G}(\mathbb{R}) \cong \mathrm{SO}(n, 1), n \geq 6 \\
n & \mathbf{G}(\mathbb{R}) \cong \mathrm{SU}(n, 1)
\end{array}\right.
$$

4.2. The prime case. Fix a prime ideal $\mathfrak{p} \subset \mathcal{O}_{F}$ not dividing $\mathfrak{d}_{1}$ and let $\mathrm{k}_{\mathfrak{p}}=\mathcal{O}_{F} / \mathfrak{p}$ denote the residue field. The group scheme $\mathbf{G}_{k_{\mathfrak{p}}}$ is then an algebraic group defined over $k_{\mathfrak{p}}$ and $\mathbf{G}\left(\mathcal{O}_{F} / \mathfrak{p}\right)=\mathbf{G}\left(k_{\mathfrak{p}}\right)$ is its set of $k_{\mathfrak{p}}$-rational points, which is a quasisimple group of Lie type. The smallest dimension of an irreducible representation of such groups was studied in [Lan72, LS74, SZ93]. We quote here the bounds in [SZ93] that are relevant to our case. 
- When $G \cong \mathrm{SO}(n, 1)$ and $n$ is even, $\mathrm{G}\left(\mathrm{k}_{\mathfrak{p}}\right) \cong \mathrm{SO}_{n+1}\left(\mathrm{k}_{\mathfrak{p}}\right)$ is the split orthogonal group of that rank over $\mathrm{k}_{\mathfrak{p}}$. If $n$ is odd, then $\mathbf{G}\left(\mathrm{k}_{\mathfrak{p}}\right) \cong \mathrm{SO}_{n+1}^{ \pm}\left(\mathrm{k}_{\mathfrak{p}}\right)$ is one of the two orthogonal groups. In these cases, the dimension of any nontrivial irreducible representation of $\mathbf{G}\left(\mathrm{k}_{\mathfrak{p}}\right)$ is bounded below by $c\left|\mathrm{k}_{\mathfrak{p}}\right|^{n-2}$ for $n \geq 6$ and by $c\left|\mathrm{k}_{\mathfrak{p}}\right|$ for $n<6$.

- When $G$ is a unitary group, either $\mathbf{G}\left(\mathrm{k}_{\mathfrak{p}}\right) \cong \mathrm{SU}_{n+1}\left(\mathrm{k}_{\mathfrak{p}}\right)$ or $\mathbf{G}\left(\mathrm{k}_{\mathfrak{p}}\right) \cong \mathrm{SL}_{n+1}$ depending on whether $\mathfrak{p}$ is inert or split in a corresponding quadratic extension of $F$ (we include the finite set of ramified primes in the idea $\mathfrak{d})$. In these cases, the dimension of any nontrivial irreducible representation of $\mathbf{G}\left(\mathrm{k}_{\mathfrak{p}}\right)$ is bounded below by $c\left|\mathrm{k}_{\mathfrak{p}}\right|^{n}$.

Consequently, we get that in all cases the representations of prime level $\mathfrak{p}$ satisfy $\operatorname{dim}(\rho) \gtrsim$ $\left|\mathrm{k}_{\mathfrak{p}}\right|^{e}$, establishing Proposition 4.2 for prime ideals.

4.3. The prime power case. We fix a prime ideal $\mathfrak{p}$ not dividing $\mathfrak{d}_{1}$ and an integer $r>1$; write $k=\left[\frac{r}{2}\right]$ for the integer part of $r / 2$. We will also assume from here on that the characteristic, $p$, of $\mathrm{k}_{\mathfrak{p}}$ does not ramify in $F$ and that it is not bad for $G$ (see [SS70, I $\S 4$ ] for details). For this we only need to exclude a finite number of primes, which we include in the ideal $\mathfrak{d}$. Under these assumptions, we will show that all irreducible representations of level $\mathfrak{p}^{r}$ satisfy

$$
\operatorname{dim}(\rho) \gtrsim\left|\mathrm{k}_{\mathfrak{p}}\right|^{2 k e}=\left\{\begin{array}{cc}
\left|\mathrm{k}_{\mathfrak{p}}\right|^{r e} & r=2 k \\
\left|\mathrm{k}_{\mathfrak{p}}\right|^{r-1) e} & r=2 k+1
\end{array}\right.
$$

Consider the finite local ring $\mathcal{O}=\mathcal{O}_{F} / \mathfrak{p}^{r}$. Slightly abusing notation, we will denote by $\mathfrak{p}=\mathfrak{p O}$ the maximal ideal of $\mathcal{O}$. Since $\mathfrak{p}$ is prime to $\mathfrak{d}_{1}, \mathcal{O}$ is a quotient of $\left(\mathcal{O}_{F}\right)_{\mathfrak{d}_{1}}$ and we may replace $\mathbf{G}$ with $\mathbf{G}_{\mathcal{O}}$ (which we denote $\mathbf{G}$ from now on). For any $\frac{r}{2} \leq l \leq r$ denote by $\mathbf{G}\left(\mathfrak{p}^{l}\right)$ the kernel of the projection from $\mathbf{G}(\mathcal{O})$ to $\mathbf{G}\left(\mathcal{O} / \mathfrak{p}^{l}\right)$. Then $\mathbf{G}\left(\mathfrak{p}^{l}\right)$ is commutative. Moreover, if $\pi \in \mathcal{O}$ is a uniformizer for $\mathfrak{p}$, then the map $I+\pi^{l} X \mapsto X$ is an isomorphism of $\mathbf{G}\left(\mathfrak{p}^{l}\right)$ with $\mathfrak{g}\left(\mathcal{O} / \mathfrak{p}^{k-l}\right)$ where $\mathfrak{g}=\operatorname{Lie}(\mathbf{G})$ (cf. [Wei84, Lemma 5.2]).

For any $X \in \mathfrak{g}(\mathcal{O})$ denote by $C_{G\left(\mathcal{O} / \mathfrak{p}^{l}\right)}(X)$ and $C_{\mathfrak{g}\left(\mathcal{O} / \mathfrak{p}^{l}\right)}(X)$ the centralizers of $X$ in $G\left(\mathcal{O} / \mathfrak{p}^{l}\right)$ and $\mathfrak{g}\left(\mathcal{O} / \mathfrak{p}^{l}\right)$ respectively. In particular, for $l=1$ we have,

$$
C_{G\left(\mathrm{k}_{\mathfrak{p}}\right)}(X)=\{g \in G(k) \mid g X=X g\}, \quad C_{\mathfrak{g}\left(\mathrm{k}_{\mathfrak{p}}\right)}(X)=\left\{Y \in \mathfrak{g}\left(\mathrm{k}_{\mathfrak{p}}\right) \mid Y X=X Y\right\} .
$$

Proposition 4.3. For any representation $\rho$ of $\mathbf{G}\left(\mathcal{O}_{F} / \mathfrak{p}^{r}\right)=\mathbf{G}(\mathcal{O})$ of level $\mathfrak{p}^{r}$ there is a nontrivial $X \in \mathfrak{g}\left(\mathrm{k}_{\mathfrak{p}}\right)$ such that

$$
\operatorname{dim}(\rho) \geq \frac{\left|\mathbf{G}\left(\mathrm{k}_{\mathfrak{p}}\right)\right|\left|\mathfrak{g}\left(\mathrm{k}_{\mathfrak{p}}\right)\right|^{k-1}}{\left|C_{\mathbf{G}\left(\mathbf{k}_{\mathfrak{p}}\right)}(X)\right|\left|C_{\mathfrak{g}\left(\mathrm{k}_{\mathfrak{p}}\right)}(X)\right|^{k-1}} .
$$

Proof. The group $\mathbf{G}\left(\mathfrak{p}^{r-k}\right)$ is a commutative normal subgroup of $\mathbf{G}(\mathcal{O})$ and the adjoint action of $\mathbf{G}\left(\mathcal{O} / \mathfrak{p}^{k}\right)=\mathbf{G}(\mathcal{O}) / \mathbf{G}\left(\mathfrak{p}^{k}\right)$ on $\mathbf{G}\left(\mathfrak{p}^{r-k}\right)$ induces a corresponding co-adjoint action on its dual $\widehat{\mathrm{G}\left(\mathfrak{p}^{r-k}\right)}$.

Let $\rho$ denote a new representation of $\mathbf{G}(\mathcal{O})$. The restriction of $\rho$ to $\mathbf{G}\left(\mathfrak{p}^{r-k}\right)$ is thus

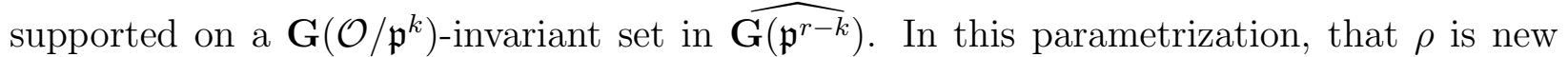
is equivalent to the restriction of $\rho$ to $\mathbf{G}\left(\mathfrak{p}^{r-1}\right)$ being non-trivial. It follows that there is at least one character $\chi \in \widehat{\mathbf{G ( \mathfrak { p } ^ { r - k } )}}$ in the support that is not trivial on $\mathbf{G}\left(\mathfrak{p}^{r-1}\right)$, and

$$
\operatorname{dim} \rho \geq \#\left\{\chi^{g} \mid g \in \mathbf{G}\left(\mathcal{O} / \mathfrak{p}^{k}\right)\right\}=\frac{\left|\mathbf{G}\left(\mathcal{O} / \mathfrak{p}^{k}\right)\right|}{\#\left\{g \in \mathbf{G}\left(\mathcal{O} / \mathfrak{p}^{k}\right) \mid \chi^{g}=\chi\right\}} .
$$


We construct an isomorphism of $\mathfrak{g}\left(\mathcal{O} / \mathfrak{p}^{k}\right)$ with $\widehat{\mathfrak{g}\left(\widehat{\mathcal{O} / \mathfrak{p}^{k}}\right)}=\widehat{\mathbf{G}\left(\mathfrak{p}^{r-k}\right)}$ that is compatible with the co-adjoint action. Let $B(X, Y)=\operatorname{tr}(\operatorname{ad}(\mathrm{X}) \operatorname{ad}(\mathrm{Y}))$ denote the Killing form on $\mathfrak{g}\left(\mathcal{O} / \mathfrak{p}^{k}\right)$. On $\mathfrak{g}(\mathcal{O} / \mathfrak{p})=\mathfrak{g}\left(\mathrm{k}_{\mathfrak{p}}\right)$, the Killing form is non-degenerate and invariant under the adjoint action. By induction, this is also true on $\mathfrak{g}\left(\mathcal{O} / \mathfrak{p}^{k}\right)$. Indeed, if there is $X \in \mathfrak{g}\left(\mathcal{O} / \mathfrak{p}^{k}\right)$ such that $B(X, Y) \equiv 0\left(\bmod \mathfrak{p}^{k}\right)$ for all $Y \in \mathfrak{g}\left(\mathcal{O} / \mathfrak{p}^{k}\right)$, then in particular it is true modulo $\mathfrak{p}$. Since over $\mathrm{k}_{\mathfrak{p}}$ the Killing form is non-degenerate, this implies that $X \equiv 0(\bmod \mathfrak{p})$ and $X=u X^{\prime}$ with $u \in \mathfrak{p}$. Dividing by $u$ we get that $B\left(X^{\prime}, Y\right) \equiv 0\left(\bmod \mathfrak{p}^{k-1}\right)$ for all $Y \in \mathfrak{g}\left(\mathcal{O} / \mathfrak{p}^{k-1}\right)$, and by induction $X \equiv 0\left(\bmod \mathfrak{p}^{k}\right)$.

Since we assume that the characteristic $p$ of $\mathrm{k}_{\mathfrak{p}}$ is unramified in $F$, the group $\mathcal{O} / \mathfrak{p}^{k} \cong$ $\left(\mathbb{Z} / p^{k} \mathbb{Z}\right)^{f}$ is a free $\mathbb{Z} / p^{k} \mathbb{Z}$ module of rank $f$ (the inertia degree). Fixing a basis, the action of multiplication by $a \in \mathcal{O} / \mathfrak{p}^{k}$ gives an embedding $\mathcal{O} / \mathfrak{p}^{k} \hookrightarrow \operatorname{Mat}\left(f, \mathbb{Z} / p^{k} \mathbb{Z}\right)$ and we denote by $\operatorname{tr}(a) \in \mathbb{Z} / p^{k} \mathbb{Z}$ the trace of the corresponding matrix. The map $\operatorname{tr}: \mathcal{O} / \mathfrak{p}^{k} \rightarrow \mathbb{Z} / p^{k} \mathbb{Z}$ is surjective and satisfies that $\operatorname{tr}(a b) \equiv 0\left(\bmod p^{k}\right)$ for all $b \in \mathcal{O} / \mathfrak{p}^{k}$ if and only if $a \equiv 0$ $\left(\bmod \mathfrak{p}^{k}\right)$. We now construct the isomorphism $\left.\mathfrak{g}\left(\mathcal{O} / \mathfrak{p}^{k}\right) \cong \mathfrak{g} \widehat{\left(\mathcal{O} / \mathfrak{p}^{k}\right.}\right)$ sending $X \in \mathfrak{g}\left(\mathcal{O} / \mathfrak{p}^{k}\right)$ to the character $\left.\chi_{X} \in \widehat{\mathfrak{g}} \widehat{\left(\mathcal{O} / \mathfrak{p}^{k}\right.}\right)$ given by $\chi_{X}(Y)=\exp \left(\frac{2 \pi i \operatorname{tr}(B(X, Y))}{p^{k}}\right)$.

With the above identification, the co-adjoint action of $\mathbf{G}\left(\mathcal{O} / \mathfrak{p}^{k}\right)$ on $\left.\mathfrak{g} \widehat{\left(\mathcal{O} / \mathfrak{p}^{k}\right.}\right)$ is identified with the adjoint action of $\mathbf{G}$ on its Lie algebra, indeed $\chi_{X}^{g}(Y)=\chi_{X}\left(g Y g^{-1}\right)=\chi_{g^{-1}}{ }_{X}(Y)$. Moreover, notice that $\chi_{X}$ is trivial on $\mathbf{G}\left(\mathfrak{p}^{r-1}\right) \cong \mathfrak{g}\left(\mathfrak{p}^{k-1} / \mathfrak{p}^{k}\right)$ if and only if $X \equiv 0(\bmod \mathfrak{p})$. Consequently, we get that there is $X \in \mathfrak{g}\left(\mathcal{O} / \mathfrak{p}^{k}\right)$ with $X \not \equiv 0(\bmod \mathfrak{p})$ such that

$$
\operatorname{dim} \rho \geq \frac{\left|\mathbf{G}\left(\mathcal{O} / \mathfrak{p}^{k}\right)\right|}{\left|C_{\mathbf{G}\left(\mathcal{O} / \mathfrak{p}^{k}\right)}(X)\right|}=\frac{\left|\mathbf{G}\left(\mathrm{k}_{\mathfrak{p}}\right)\right|\left|\mathfrak{g}\left(\mathrm{k}_{\mathfrak{p}}\right)\right|^{k-1}}{\left|C_{\mathbf{G}\left(\mathcal{O} / \mathfrak{p}^{k}\right)}(X)\right|} .
$$

Finally, we can write

$$
\left|C_{\mathbf{G}\left(\mathcal{O} / \mathfrak{p}^{k}\right)}(X)\right|=\sum_{a \in C_{\mathbf{G}\left(\mathcal{O} / \mathfrak{p}^{k-1}\right)}(X)} \#\left\{g \in C_{\mathbf{G}\left(\mathcal{O} / \mathfrak{p}^{k}\right)}(X) \mid g \equiv a \quad\left(\bmod \mathfrak{p}^{k-1}\right)\right\} .
$$

If $g_{1}, g_{2} \in C_{\mathbf{G}\left(\mathcal{O} / \mathfrak{p}^{k}\right)}(X)$ and $g_{1} \equiv g_{2}\left(\bmod \mathfrak{p}^{k-1}\right)$ then $g_{1} g_{2}^{-1}=I+\pi^{k-1} Y$ with $Y \in C_{\mathfrak{g}(\mathcal{O} / \mathfrak{p})}(X)$. Hence, the number of such elements for each $a$ is bounded by $\left|C_{\mathfrak{g}\left(\mathrm{k}_{\mathfrak{p}}\right)}(X)\right|$ and by induction we find that $\left|C_{\mathbf{G}\left(\mathcal{O} / \mathfrak{p}^{k}\right)}(X)\right| \leq\left|C_{\mathbf{G}\left(\mathrm{k}_{\mathfrak{p}}\right)}(X)\right|\left|C_{\mathfrak{g}\left(\mathrm{k}_{\mathfrak{p}}\right)}(X)\right|^{k-1}$ concluding the proof.

Since $\left|\mathbf{G}\left(\mathrm{k}_{\mathfrak{p}}\right)\right| \gtrsim\left|\mathrm{k}_{\mathfrak{p}}\right|^{\operatorname{dim}(G)}$ and $\left|\mathfrak{g}\left(\mathrm{k}_{\mathfrak{p}}\right)\right|=\left|\mathrm{k}_{\mathfrak{p}}\right|^{\operatorname{dim}(G)}$, the bound (4.2) follows from the following estimate on the cardinality of centralizers.

Proposition 4.4. Let $X \in \mathfrak{g}\left(\mathrm{k}_{\mathfrak{p}}\right)$ denote a nontrivial element. Then,

$$
\left|C_{\mathfrak{g}\left(\mathrm{k}_{\mathfrak{p}}\right)}(X)\right| \leq\left|\mathrm{k}_{\mathfrak{p}}\right|^{\operatorname{dim}(G)-2 e} \text { and }\left|C_{\mathbf{G}\left(\mathrm{k}_{\mathfrak{p}}\right)}(X)\right| \lesssim\left|\mathrm{k}_{\mathfrak{p}}\right|^{\operatorname{dim}(\mathbf{G})-2 e}
$$

Proof. Let $G=\mathbf{G}_{\mathrm{k}_{\mathfrak{p}}}$, so that $G$ is one of the groups $\mathrm{SL}_{n+1}, \mathrm{SU}_{n+1}$ or $\mathrm{SO}_{n+1}^{ \pm}$over the finite field $\mathrm{k}_{\mathfrak{p}}$, and let $\mathfrak{g}=\operatorname{Lie}(G)$ be its Lie algebra. Let $\overline{\mathrm{k}}_{\mathfrak{p}}$ be an algebraic closure of $\mathrm{k}_{\mathfrak{p}}$. Then $G=G\left(\bar{k}_{\mathfrak{p}}\right)$ is an algebraic group defined over $\mathrm{k}_{\mathfrak{p}}$ and for any $X \in \mathfrak{g}\left(\mathrm{k}_{\mathfrak{p}}\right)$, the centralizer $C_{G}(X)$ is an algebraic subgroup of $G$ whose $\mathrm{k}_{\mathfrak{p}}$ points are precisely $C_{G\left(\mathrm{k}_{\mathfrak{p}}\right)}(X)$. Moreover, by $\left[\mathrm{SS70}\right.$, Corollary 5.2] we have that $C_{\mathfrak{g}}(X)$ is the Lie algebra of $C_{G}(X)$.

It is known that the number of $\mathbf{k}_{\mathfrak{p}}$-rational points of any connected algebraic group $H$ defined over $k_{\mathfrak{p}}$ is roughly $\left|k_{\mathfrak{p}}\right|^{\operatorname{dim} H}-$ a precise bound is

$$
\left(\left|\mathrm{k}_{\mathfrak{p}}\right|-1\right)^{\operatorname{dim}(H)} \leq\left|H\left(\mathrm{k}_{\mathfrak{p}}\right)\right| \leq\left(\left|\mathrm{k}_{\mathfrak{p}}\right|+1\right)^{\operatorname{dim} H},
$$


(cf. [Nor87, Lemma 3.5]). The group $C_{G}(X)$ is not necessarily connected. However, by [SS70, Chapter II, 4.1 and 4.2]) and [SS70, Chapter IV, 2.26] its number of connected components is uniformly bounded. Consequently, to bound the size of the centralizer it is sufficient to bound the dimension, that is, to show

$$
\operatorname{dim}\left(C_{\mathfrak{g}}(X)\right) \leq \operatorname{dim}(G)-2 e .
$$

We will show that this holds for any $X \in \mathfrak{g}\left(\overline{\mathrm{k}}_{\mathfrak{p}}\right)$; the statement is now purely about the algebraic group $G$ defined over an algebraically closed field.

Recall the Jordan decomposition in the Lie algebra; for any $X \in \mathfrak{g}$ there is a unique decomposition $X=X_{s}+X_{n}$ with $X_{s}$ semi-simple and $X_{n}$ nilpotent which commute with each other. Since this decomposition is unique, $C_{G}(X)=C_{G}\left(X_{s}\right) \cap C_{G}\left(X_{n}\right)$. Consequently, we may assume that $X$ is either semisimple or nilpotent.

We start with $G=\mathrm{SL}_{n+1}$ (corresponding to $\mathbf{G}(\mathbb{R})$ being a unitary group) so that $\operatorname{dim} G=$ $n^{2}+2 n$ and $e(G)=n$. Let $X \in \mathfrak{g}$ denote a non-trivial semi-simple element. We may assume that $X$ lies in the Lie algebra of the standard split torus, in which case it is easy to determine the centralizer explicitly. A simple computation shows that if $r_{1}, \ldots, r_{d}$ are the multiplicities of the $d$ distinct eigenvalues of $X$, so that $r_{1}+\ldots+r_{d}=n+1$, then

$$
\operatorname{dim} C_{\mathfrak{g}}(X)=\sum_{j=1}^{d} r_{d}^{2}-1
$$

This is clearly maximal when there are only two eigenvalues of multiplicities $r_{1}=1$ and $r_{2}=n$ respectively, so that

$$
\operatorname{dim} C_{\mathfrak{g}}(X) \leq n^{2} \leq \operatorname{dim} G-2 e
$$

Next, let $X \in \mathfrak{g}$ be a nontrivial nilpotent element and let $r_{j}, j=1, \ldots, d$ denote the number of blocks of size $j$ in its Jordan normal form. Then $\sum_{j} j r_{j}=n+1$ and

$$
\operatorname{dim} C_{\mathfrak{g}}(X)=\sum_{j=1}^{d}\left(r_{j}+\ldots+r_{d}\right)^{2}-1,
$$

(see [SS70, Chapter IV, 1.8]). This is maximal when $r_{1}=n-1$ and $r_{2}=1$ so that

$$
\operatorname{dim} C_{\mathfrak{g}}(X) \leq n^{2}-1 \leq \operatorname{dim}(G)-2 e .
$$

We consider the orthogonal group, $G=\mathrm{SO}_{n+1}$ with its canonical inclusion in $\mathrm{SL}_{n+1}$. We have $\operatorname{dim}(G)=\frac{n(n+1)}{2}$ as well as $e=(n-2)$ for $n \geq 6$ and $e=1$ for $n<6$. Let $X \in \mathfrak{g}$ be a semisimple element. Since $X^{t}=-X$, the nonzero eigenvalues of $X$ come in pairs $\lambda,-\lambda \in \overline{\mathrm{k}}_{\mathfrak{p}}^{\times}$each having the same multiplicity. Let $r_{0}=\operatorname{dim} \operatorname{ker}(X)$ and let $r_{1}, \ldots, r_{d}$ denote the multiplicities of distinct pairs of nonzero eigenvalues, so that $r_{0}+2\left(r_{1}+\ldots+r_{d}\right)=n+1$. For such an element, we can compute the centralizer explicitly, and its dimension is given by

$$
\operatorname{dim} C_{\mathfrak{g}}(X)=\frac{r_{0}\left(r_{0}-1\right)}{2}+\sum_{j=1}^{d} r_{j}^{2}
$$

Clearly, this is maximal if there is only one pair of nonzero eigenvalues (with multiplicity $r_{1}$ ) and the zero eigenvalue with multiplicity $r_{0}=n+1-2 r_{1}$, in which case the dimension is

$$
\operatorname{dim} C_{\mathfrak{g}}(X)=\frac{(n+1) n}{2}+r_{1}\left(3 r_{1}-2 n-1\right)
$$


For $n \neq 3,5$ this is maximal when $r_{1}=1$ and

$$
\operatorname{dim} C_{\mathfrak{g}}(X) \leq \frac{(n+1) n}{2}-2(n-1) \leq \operatorname{dim}(G)-2 e
$$

When $n=3,5$ the maximal dimension is obtained when $r_{1}=\frac{n+1}{2}$ and it is 4 and 9 respectively, in particular it is bounded by $\operatorname{dim}(G)-2$.

Finally, for a non-trivial nilpotent element $X \in \mathfrak{g}\left(\overline{\mathrm{k}}_{\mathfrak{p}}\right)$, again write it in its Jordan normal form (see [SS70, IV, §2.19] for the normal form in the orthogonal group). Denote by $r_{j}$ the number of blocks of size $j$, then $\sum j r_{j}=n+1$ and $r_{j}$ is even for even $j$. With this data we have

$$
\operatorname{dim} C_{\mathfrak{g}}(X)=\frac{1}{2} \sum_{j}\left(r_{j}+\ldots+r_{d}\right)^{2}-\frac{1}{2} \sum_{j \text { odd }} r_{j}
$$

It is not hard to see that this is maximal when $r_{1}=n-2$ and $r_{3}=1$ so that

$$
\operatorname{dim} C_{\mathfrak{g}}(X) \leq \frac{n(n+1)}{2}-2(n-1) \leq \operatorname{dim}(G)-2 e
$$

To conclude, Propositions 4.3 and 4.4 give the bound (4.2). We thus get that a representation $\rho$ of level $\mathfrak{p}^{r}$ satisfies $\operatorname{dim}(\rho) \gtrsim\left|k_{\mathfrak{p}}\right|^{e r}$ for even $r$, and $\operatorname{dim}(\rho) \gtrsim\left|k_{\mathfrak{p}}\right|^{e(r-1)} \geq\left|k_{\mathfrak{p}}\right|^{2 r e / 3}$ for odd $r \geq 3$. Together with the prime case discussed above, this concludes the proof of Proposition 4.2, and hence of Theorem 4 .

\section{REFERENCES}

[BB10] V. Blomer and F. Brumley, On the Ramanujan conjecture over number fields. Preprint, arXiv:math.NT/1003.0559, 2010.

[BGS10a] J. Bourgain, A. Gamburd and P. Sarnak, Affine linear sieve, expanders, and sum-product. Invent. Math. 179, No. 3., (2010), 559-644.

[BGS10b] _ Generalization of Selberg's 3/16 theorem and affine sieve. Invent. Math. (to appear).

[BKS10] J. Bourgain, A. Kontorovich, and P. Sarnak, Sector estimates for hyperbolic isometries. Preprint arXiv:math.NT/1001.4541, (2010).

[BV10] J. Bourgain and P. P. Varjú, Expansion in $\mathrm{SL}_{\mathrm{d}}(\mathbb{Z} / \mathrm{q} \mathbb{Z})$, q arbitrary. Preprint, arXiv:math.GR/1006.3365, 2010.

[BGT10] E. Breuillard, B. Green, and T. Tao, Approximate subgroups of linear groups. Preprint, arXiv:math.GR/1005.1881, (2010).

[Bro85] R. Brooks, The first eigenvalue in a tower of coverings. Bull. Amer. Math. Soc. (N.S.) 13 (1985), no. 2, 137-140. MR 799796

[BS91] M. Burger and P. Sarnak, Ramanujan duals. II Invent. Math. 106 (1991), no. 1, 1-11.

[Clo03] L. Clozel, Démonstration de la conjecture $\tau$. Invent. Math. 151 (2003), no. 2, 297-328.

[dGW78] D. L. de George and N. R. Wallach, Limit formulas for multiplicities in $L^{2}(\Gamma \backslash G)$. Ann. of Math. (2) 107 (1978), no. 1, 133-150. MR 0492077

[GN09] A. Gorodnik and A. Nevo, Counting lattice points. Preprint, arXiv:math.DS/0903.1515, 2009.

[HK93] J. Huntley and Y. R. Katznelson, Density theorems for congruence groups in real rank 1 . Duke Math. J. 71 (1993), no. 2, 463-473. MR 1233445

[Kel10] D. Kelmer, A uniform strong spectral gap for congruence covers of a compact quotient of PSL $(2, \mathbb{R})^{\mathrm{d}}$. Int. Math. Res. Not. (2010).

[KS09] D. Kelmer and P. Sarnak, Strong spectral gaps for compact quotients of products of PSL(2, $\mathbb{R})$. J. Eur. Math. Soc. 11 (2009), no. 2, 283-313. 
[KS03] H. H. Kim and Peter Sarnak, Refined estimates towards the Ramanujan and Selberg conjectures. Appendix to H. H. Kim, J. Amer. Math. Soc. 16 (2003), no. 1, 139-183.

[Kna86] A. W. Knapp, Representation theory of semisimple groups. Princeton Mathematical Series, vol. 36, Princeton University Press, Princeton, NJ, 1986, An overview based on examples. MR 855239

[Lan72] V. Landázuri, Bounds for the characters of the Chevalley groups. Rev. Colombiana Mat. 6 (1972), 125-164. MR 0379686

[LS74] V. Landazuri and G. M. Seitz, On the minimal degrees of projective representations of the finite Chevalley groups. J. Algebra 32 (1974), 418-443. MR 0360852

[Lub10] A. Lubotzky, Discrete groups, expanding graphs and invariant measures. Modern Birkhäuser Classics, Birkhäuser Verlag, Basel, 2010, With an appendix by Jonathan D. Rogawski, Reprint of the 1994 edition. MR 2569682

[LZ03] A. Lubotzky and A. Żuk, On property $(\tau)$. Book in preparation; draft available at http://math.huji.ac.il/ alexlub, 2003.

[LRS99] W. Luo, Z. Rudnick, and P. Sarnak, On the generalized Ramanujan conjecture for GL( $n)$. Automorphic forms, automorphic representations, and arithmetic (Fort Worth, TX, 1996), Proc. Sympos. Pure Math., vol. 66, Amer. Math. Soc., Providence, RI, 1999, pp. 301-310. MR 1703764

[Nor87] M. V. Nori, On subgroups of $\mathrm{GL}_{n}\left(\mathbf{F}_{p}\right)$. Invent. Math. 88 (1987), no. 2, 257-275. MR 880952

[PS10] L. Pyber and E. Szabó, Growth in finite simple groups of Lie type of bounded rank. preprint, arXiv:math.GR/1005.1858, 2010.

[Ran74] B. Randol, Small eigenvalues of the Laplace operator on compact Riemann surfaces. Bull. Amer. Math. Soc. 80 (1974), 996-1000. MR 0400316

[SV10] A. Salehi Golsefidy and P. P. Varjú, Expansion in perfect groups. In preparation.

[SX91] P. Sarnak and X. X. Xue, Bounds for multiplicities of automorphic representations. Duke Math. J. 64 (1991), no. 1, 207-227.

[Sel65] A. Selberg, On the estimation of Fourier coefficients of modular forms. Proc. Sympos. Pure Math., Vol. VIII, Amer. Math. Soc., Providence, R.I., 1965, pp. 1-15.

[SS70] T. A. Springer and R. Steinberg, Conjugacy classes. Seminar on Algebraic Groups and Related Finite Groups (The Institute for Advanced Study, Princeton, N.J., 1968/69), Lecture Notes in Mathematics, Vol. 131, Springer, Berlin, 1970, pp. 167-266. MR 0268192

[SZ93] G. M. Seitz and Alexander E. Zalesskii, On the minimal degrees of projective representations of the finite Chevalley groups. II J. Algebra 158 (1993), no. 1, 233-243. MR 1223676

[Var10] P. P. Varjú, Expansion in \$SL_d(O_K/I)\$, \$I square-free. preprint, arXiv:math.GR/1001.3664, 2010.

[Wei84] B. Weisfeiler, Strong approximation for Zariski-dense subgroups of semisimple algebraic groups. Ann. of Math. (2) 120 (1984), no. 2, 271-315. MR 763908

[Xue91] X. X. Xue, On the first Betti numbers of hyperbolic surfaces. Duke Math. J. 64 (1991), no. 1, 85-110. MR 1131394

Department of Mathematics, University of Chicago, 5734 S. University Avenue Chicago, ILLINOIS 60637

E-mail address: kelmerdu@math.uchicago.edu

University of British Columbia, Department of Mathematics 1984 Mathematics Road VanCOUVER BC V6T $1 \mathrm{Z} 2$

E-mail address: lior@math.ubc.ca 